\title{
Approaching the structure of human VDAC1, a key molecule in mitochondrial cross-talk
}

\author{
Kornelius Zeth • Thomas Meins • Clemens Vonrhein
}

Published online: 9 August 2008

(C) Springer Science + Business Media, LLC 2008

\begin{abstract}
The voltage dependent anion-channel, VDAC, is the major constitutive protein of the outer membrane of mitochondria. Functionally, VDAC is involved in the exchange of small metabolites over the mitochondrial outer membrane and supports enzymes of the cytoplasm with energy precursors i.e. ATP. Moreover, the channel alone or in complex with proteins of the inner mitochondrial membrane or the intermembrane space provides a basis for docking of cytosolic proteins which can regulate outer membrane permeability in several ways. Structurally, this channel has a bacterial origin by evolution and partly resembles bacterial porin functions. However, the structure seems more complex as a variety of interactions on both channel sides can occur. Therefore, our work described is aiming to determine the structure of VDAC at atomic resolution and together with functional data to understand better how this channel can carry out such a variety of differing functions.
\end{abstract}

\footnotetext{
K. Zeth $(\bowtie)$

Department of Protein Evolution,

Max Planck Institute for Developmental Biology,

Spemannstr. 35,

72076 Tübingen, Germany

e-mail: kornelius.zeth@tuebingen.mpg.de

T. Meins

Department of Membrane Biochemistry,

Max Planck Institute of Biochemistry,

Am Klopferspitz 18,

82152 Martinsried, Germany

C. Vonrhein

Global Phasing Ltd.,

Sheraton House, Castle Park,

Cambridge CB3 OAX, UK
}

Keywords VDAC $\cdot$ HVDAC1 1 Structure . Electron density $\cdot$ Crystals

\section{Introduction}

The outer membrane of mitochondria harbors four integral membrane proteins or membrane protein complexes, respectively. This finding is in strong contrast to a typically large number of proteins predicted to exist in the outer membrane of most Gram-negative bacteria, which are related to mitochondria through the endosymbiotic theory. Even with this small subset of proteins it enables the full communication between mitochondria and the cellular environment. Interestingly, three of these mitochondrial outer membrane complexes (the general import pore TOM, the SAM and MDM10 complexes) allow for the entire translocation and insertion of all newly synthesized proteins destined to the mitochondrial organelle (Bolender et al. 2008). The fourth protein family of voltage dependent anion-channels (VDACs) was initially described as being reminiscent of bacterial porins and to be mainly responsible for the exchange of chemical energy from the cytosol to the mitochondrion and vice versa (Bowen et al. 1985; Guo et al. 1995). This subset of channel proteins typically occurs with a high sequence homology between unicellular and higher eukaryotes and a sequence identity of $>80 \%$ between up to three isoforms which are observed in mammals. Among all mitochondria studied by semi-quantitative methods this class of proteins is the most abundant specimen found in the MOM (Mannella 1998a, b). In human three different isoforms HVDAC1-HVDAC3 with high sequence analogy $(>80 \%)$ exist according to the genome (Young et al. 2007). However, these isoforms are not only regulated in their relative abundance in differing cell types but they can 
also be found in phosphorylated states at certain stages of the cell cycle and specific functions can thereby be stimulated accordingly (Schwertz et al. 2007).

VDAC from Saccharomyces cerevisiae and Neurospora crassa were the first VDAC proteins isolated and studied by various biophysical techniques. However, first structural snapshots at medium resolution were derived from HVDAC 1 and VDAC from $N$. crassa by electron microscopy of 2D crystals (Mannella 1998a, b; Dolder et al. 1999). Together with single channel conductance methods used to characterize this protein channel the passive diffusion of small metabolites in mitochondria was tested and the protein and was treated in function similarly to the related bacterial porins. However, several years ago this functional view turned out to be insufficient since in fungi a number of interactions between VDAC and e.g. the ANT of the inner membrane are said to be missing in the bacterial precursors (Komarov et al. 2005). Among these functions the involvement in coupling mitochondria with energy maintenance is the most prominent function described so far (Pastorino and Hoek 2003). For the three isoforms appearing in mammals a variety of interactions between these proteins have been described on both sides of the membrane placing VDAC proteins in the center of the mitochondrial communication (Brdiczka et al. 1998).

While interactions with the inter membrane space of mitochondria are typically related to energy metabolism, the interplay with the cytosol appears to be even more complex. VDAC is described to be involved in interactions with a growing number of proteins, most of which are involved in the mitochondrial phase of apoptosis. Initially, a supramolecular pore channel (also termed the permeability transition pore, PTP) including VDAC and ANT from the inner membrane spanning both membranes was proposed to be regulated by factors which may lead to the breakdown of the inner membrane potential (Bernardi and Forte 2007; Grimm and Brdiczka 2007). However, another way of describing the active functioning of VDAC in related apoptotic pathways emerges and it appears that more and more additional functions may be addressed. Here, HVDAC1 was described to anchor proteins e.g. tBid or Bcl-xl to the outer membrane and to induce mitochondrial apoptosis by a controlled multi-step process (Rostovtseva et al. 2004; Malia and Wagner 2007). By contrast isoform II has been described to directly bind the pro-apoptotic member Bak and to potentially facilitate insertion and association of Bak into pores which can release mitochondrial cyt $\mathrm{c}$ that will induce downstream apoptosis (Cheng et al. 2003). Recently, the attachment of the lipid synthesis protein STAR to VDAC during the life cycle of a eukaryotic cell was described (Bose et al. 2008). Other reports depict VDAC as part of a tRNA uptake system in plant cells (Salinas et al. 2006).
In recent years several reports described the mitochondrial VDAC as a potential target for pharmaceutical research. Several drugs were described that potentially bind to VDAC as their target molecule in vivo. As a basis for a more thorough understanding of the functional role of VDAC alone and VDAC-complexes in biology and to allow further target screening of VDAC using the most prominent isoform I from human we set out to structurally characterize the protein by biophysical techniques including electron microscopy and X-ray crystallography.

\section{Characterization of recombinant HVDAC1}

In our lab the human isoforms I and II were over-expressed in Escherichia coli as insoluble inclusion bodies and a refolding procedure was established that allowed producing quantities of around $100 \mathrm{mg}$ of both isoforms within 1 week. The correct fold of the protein was initially tested using secondary structure assignment methods like FTIRand CD-spectroscopy (see Fig. 1a,b). Both methods clearly indicated proper folding of the target protein yielding an approximate overall content of $46 \% \beta$-sheet structure and a $\alpha$-helical contribution of $10 \%$. These values are in good agreement with values determined for VDAC proteins from fungi and a more closely related VDAC from plants (Shao et al. 1996; Koppel et al. 1998; Desai et al. 2006).

Another way of testing the correct fold of an outer membrane protein is to determine a possible gel shift in SDS-gels under non-denaturing conditions. These experiments have proven to be quite successful in the case of the bacterial outer membrane proteins (Sukumaran et al. 2006; Minetti et al. 1997). However, for the eukaryotic porin and homologs of the mitochondrial outer membrane this method failed probably because temperature transition of the $\beta$-barrel to the unfolded state already occurred at around 40 degrees (for HVDAC1: data obtained from CD-melting curves, data not shown). Nevertheless, cross-linking experiments of the recombinantly produced and refolded protein yielded both monomeric and a significant amount of dimeric species in SDS-gels. This may be taken as another indicator of proper folding since apparently oligomerization of the protein occurred (see Fig. 1c).

\section{Complexes of HVDAC1 with the pro-apoptotic protein cBid}

Other ways to test for the correct fold of a protein are looking for enzymatic activity or the ability to interact with binding partners described as in the literature (Rostovtseva et al. 2004). An interaction between HVDAC1 and cBid is described in the literature based on electrophysiological 
a

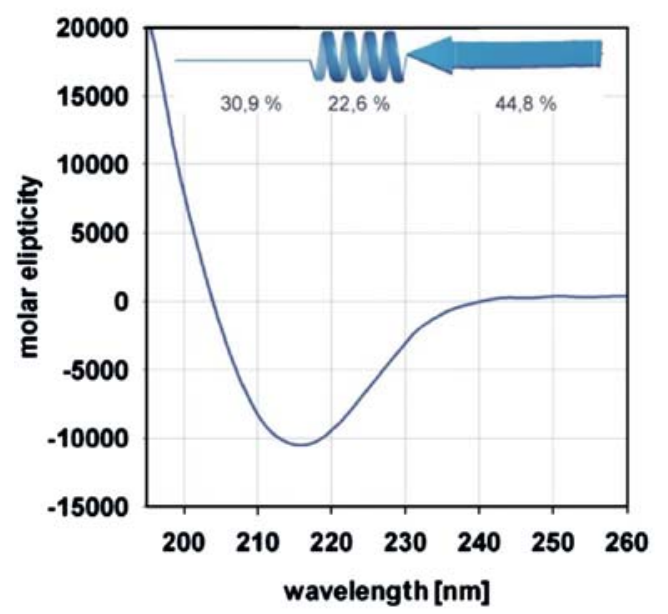

b

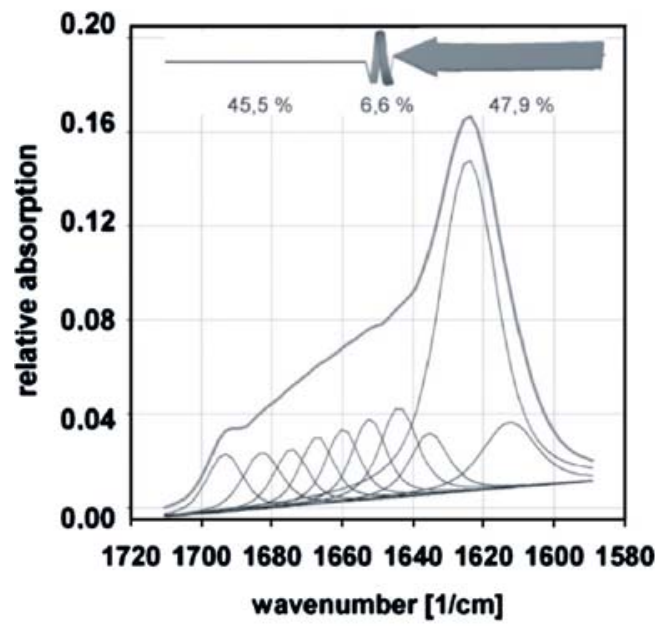

C

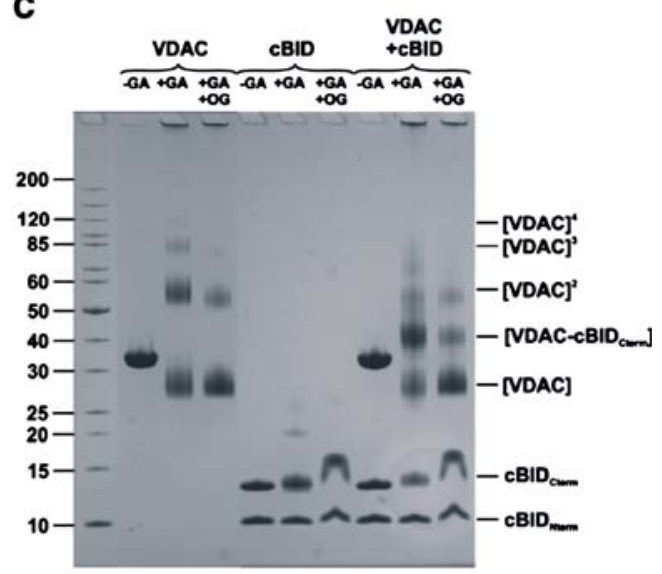

measurements and this seems at present the most reliable system to study. However, a direct interaction of both proteins has never been proven physically. In order to be able to study such complexes by X-ray crystallographic techniques we have purified both protein compounds to
Fig. 1 Secondary structure assignment of HVDAC1 and interaction studies with human cBid. a CD-spectra of HVDAC1 determined in $1 \%$ of $\beta$-octyl-glucoside as the detergent. The spectrum shows a $\beta$ structure contribution of approx. $45 \%$ plus an overestimated fraction of about $22 \% \alpha$-helix. b FTIR-spectrum of human VDAC1 in $0.8 \%$ C8E4 as the detergent. The $\beta$-sheet structure was calculated to be $48 \%$, while the $\alpha$-helical contribution is approx. $7 \%$. c Cross-link experiments with human VDAC1, alone, isolated human $\mathrm{cBid}$, and a 1:1 mixture of both proteins in the presence of glutar aldehyde. A significant portion of HVDAC1 (VDAC + GA) was cross-linked to yield a strong dimer band plus additional weak band of trimers. Interestingly, the cross-linked monomer runs at a lower molecular weight of less than $30 \mathrm{kD}$ probably due to cross-link reactions between adjacent strands of the barrel or a helix-barrel crosslink, respectively. Cross-linking between the two fragments of cBid (cBID) after caspase treatment does not occur between the two domains. However, conformational changes occur in cBid upon addition of $\beta$-octyl-glucoside and glutar aldehyde to yield a higher molecular weight specimen $(\mathrm{cBID}+\mathrm{GA}+\mathrm{OG})$. Cross-linking of VDAC and $\mathrm{cBid}(\mathrm{VDAC}+\mathrm{cBID}+\mathrm{GA}$ ) yielded a distribution of dominant 1:1 complexes and a small amount of 1:2 and 2:2 specimen. A small shift of the equilibrium towards the monomeric VDAC might be envisaged (VDAC $+\mathrm{cBID}+\mathrm{GA})$

check the possible interaction between HVDAC1 and cBid by cross-linking using glutar aldehyde and can demonstrate an interaction on SDS-PAGE. A 1:1 complex of VDAC and cBid is clearly visible as well as a smaller fraction of the hetero-trimeric complex (VDAC:cBid-2:1 ratio, Fig. 1c). A potential influence of $\mathrm{cBid}$ on the monomer-dimer equilibrium might be envisaged.

\section{Electron microscopy of HVDAC1}

Electron microscopy of membrane proteins embedded into a lipid bilayer often results in data to medium or close-to-atomic resolution. In order to achieve such a resolution we transferred VDAC into lipid bilayer and determined electron micrographs of such vesicles. Although the order of the protein was good and data to a resolution of approx. $8 \AA$ could be obtained for laterally arranged protein aggregates, the conclusion to be drawn from these experiments is rather limited since only multi-layered crystals could be obtained during this analysis. In order to characterize the molecular surface of these 2D-crystal arrays and to obtain a complementary view the crystals were shadowed from a certain angle of incidence with a thin layer of heavy atoms and those images allowed the identification of the shape of a single protein specimen. From these images it seems likely that the protein forms a dimer which is consistent with results from cross-linking and X-ray crystallography. The pore diameter was estimated to be between 4 and $5 \mathrm{~nm}$ depending on which of the pore sides was chosen (Fig. 2). 
Fig. 2 Electron micrographs of human VDAC1. Diffraction pattern computed based on 2Dcrystals of VDAC embedded in vitreous ice and freeze dried and shadowed (c-e), respectively. a Diffraction spots are visible up to 8.2 A resolution (see white circle) with a scale bar of $0.6 \mathrm{~nm}^{-1}$. b The vectors of the reciprocal space are marked with $a$ and $b$. The low density lumens are surrounded by rims of higher density (see arrows) and yield an approximate diameter of three nanometers. c Three dimensional perspective view of freeze dried and shadowed crystals calculated from six single images with different azimuths and an elevation angle of $65^{\circ}$ (scale bar $5 \mathrm{~nm}$ ). Protrusions are marked with asterisks. d Top view of the reconstructed surface (scale bar $5 \mathrm{~nm}$ ). e Correlation average of the rotary-shadowed crystal (scale bar $5 \mathrm{~nm}$ ) (with permission to reproduce from Elsevier Limited)
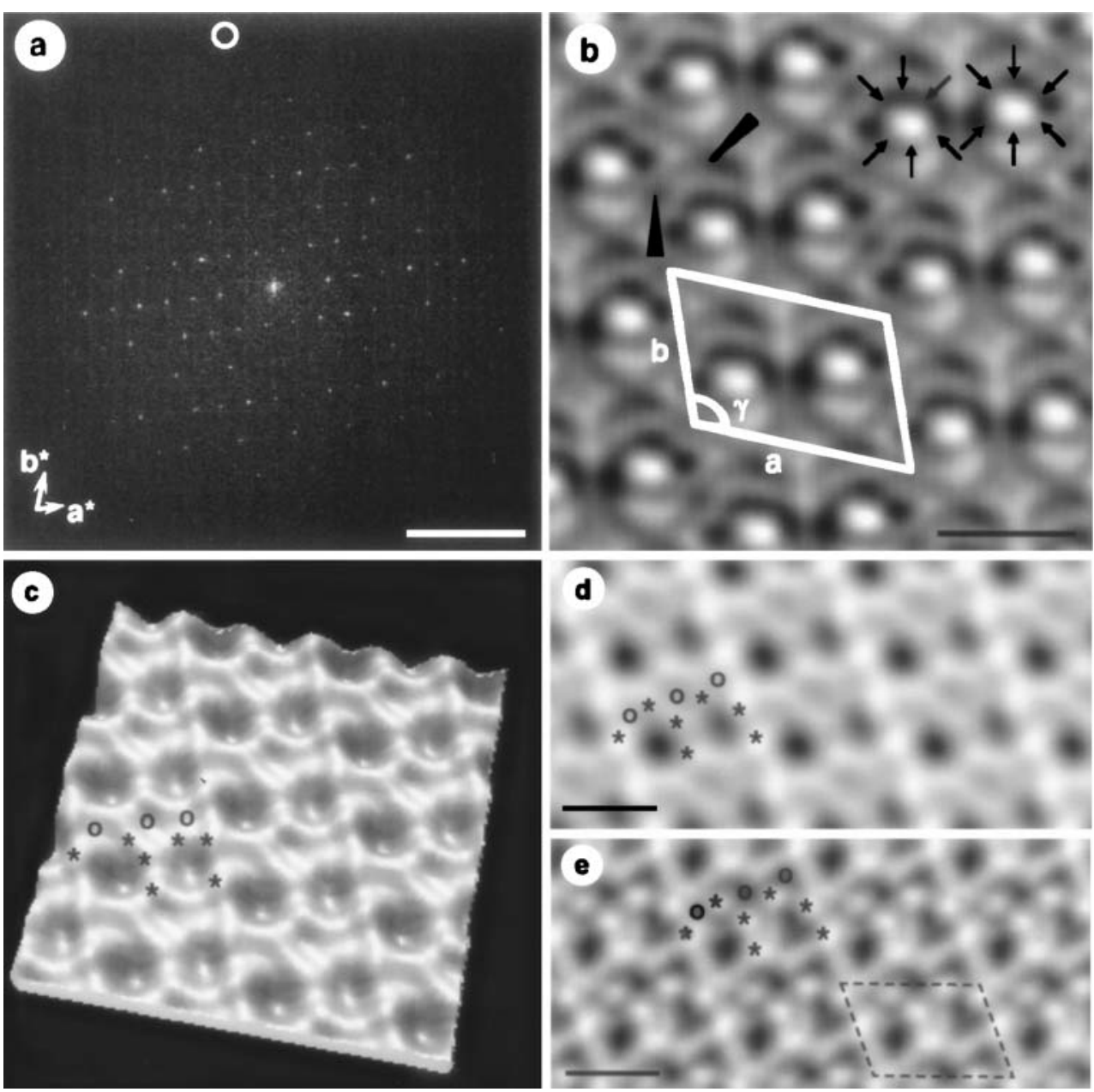

\section{The structure of HVDAC1 at higher resolution}

To obtain a picture of the channel at higher resolution we used our protein preparation method for the crystallization and screening of 3D-crystals diffracting to atomic resolution. Testing of different detergents lead to initial crystals which often diffracted to not better than $20 \AA$ resolution. Using Cymal-5 as detergent this resolution could be dramatically improved leading to crystals diffracting to $3.5 \AA$ resolution. Although this resolution in principle should allow a protein model to be built within a good quality electron density map, experimental phasing of the crystals was so far hampered by several factors including the low number of natively present methionines for SeMetphasing and the relatively poor diffraction limit. Using initial phases based on SeMet-doped crystals and multiple anomalous dispersion (MAD) techniques together with Ptbased multiple isomorphous replacement an electron density with phases up to $6 \AA$ was derived that showed the barrel wall and a number of $\beta$-strands together with the $\mathrm{N}$ terminal $\alpha$-helix in a clear view. Moreover, the dimeric origin of the protein complex by rather small proteinprotein interfaces can be seen easily. Details of the structure are currently not available since phase information to higher resolution is still missing (Fig. 3).

\section{Biological implications and outlook}

VDACs in unicellular eukaryotes i.e. S. cerevisiae differ in the complexity of interactions they are involved in as part of the entire cell. Although an apoptotic pathway in yeast has been delineated in the literature, an involvement of VDAC isoforms in this pathway could not be addressed (Mazzoni and Falcone 2008). By contrast in multi-cellular organisms and with the evolution of sophisticated apoptotic mechanisms the mitochondrial control over the fate of a certain cell became more and more evident. Complexes of VDAC with apoptotic factors i.e. cBid or Bak are of biological importance as these factors among others can stimulate a decision about life or death of a certain cell. To control this process more tightly medical treatment may help in certain diseases where cellular life is controlled in a too strict and abnormal way. Such phenomena are found among neuron-degenerative disorders and a pharmacological intervention into the interplay of VDAC and apoptotic 
A

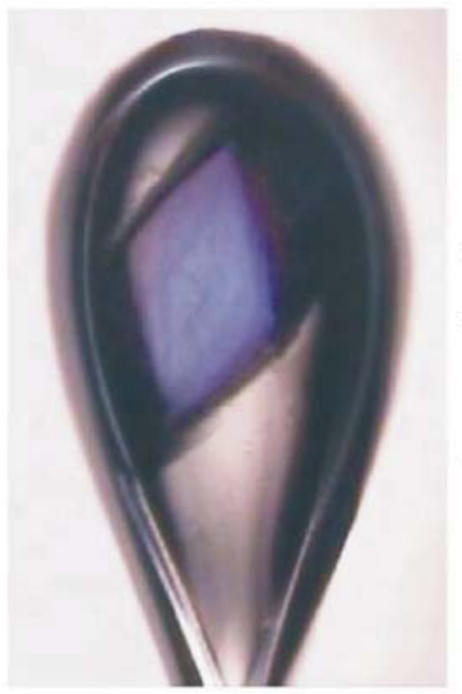

B

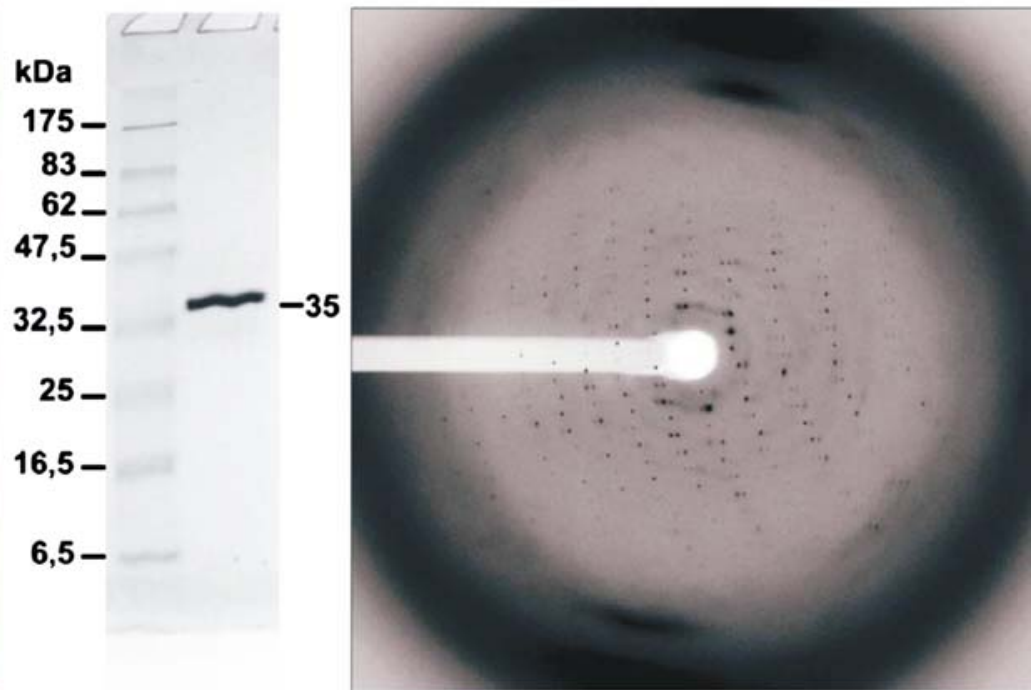

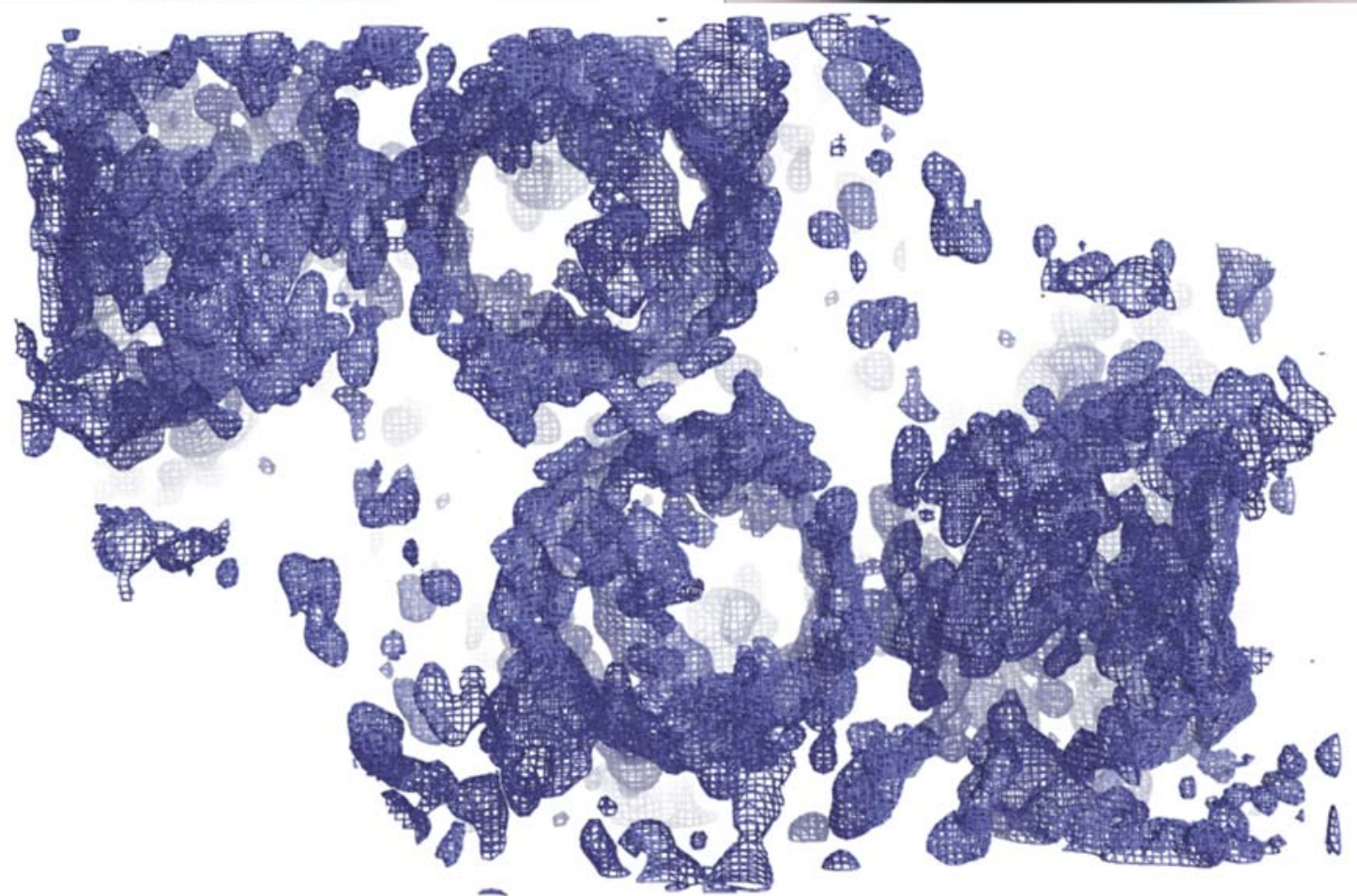

Fig. 3 Crystallization and crystallographic analysis of HVDAC1 crystals - from the crystal to the electron density and model. a Crystal of the protein embedded in a loop kept in the mother liquor and frozen in liquid nitrogen. b SDS-gel of this protein crystal dissolved in water demonstrated a single protein band which was later identified as human VDAC1 by MALDI-MS techniques and N-terminal sequenc-

factors might prolong the cellular life. However, also VDAC alone may play a more important role not only as adaptor for apoptotic factors but also as part of the permeability transition pore complex. This complex can potentially-after breakdown of the inner membrane potential - also independently of apoptotic factors initiate membrane permeability for certain mitochondrial factors. ing. c Diffraction pattern of HVDAC1 taken at the beamline PXII of the synchrotron light source SLS, Villigen, Switzerland. d Electron density of the protein based on MAD-phases (from three SeMet scatterers of the protein calculated at $6 \AA$ resolution). The dimeric structure of the protein, the diameter of approx. $3 \mathrm{~nm}$ and the localization of the N-terminal helix within the barrel are visible

Direct interactions between VDAC and potential drugs have been demonstrated over the past few years. A direct interaction between VDAC and the anti-depressant fluoxetine has been shown but a biological function of such a complex is still questionable (Nahon et al. 2006). Moreover, very recently HVDAC2 has been shown to be a potential target of erastin, a small compound which turned 
out to be a potential inhibitor specific to cancer cells (Yagoda et al. 2007). The disruption of the HVDAC1hexokinase complex has been demonstrated as a potential way of treating tumors using the plant hormone methyl jasmonate (Galluzzi et al. 2008).

Our current work is aiming to solve the structure of HVDAC1 at the resolution given and to improve the crystals quality to attain higher resolution, which is a prerequisite to better understand not only the channel architecture but may also allow to define substrate and target complexes in the near future.

Acknowledgements The author is grateful for financial support from DFG via research grants ZE522-3/3. Part of the work described has been done at the Max Planck Institute of Biochemistry in the department of Membrane Biochemistry. The support of the director Prof. Dieter Oesterhelt and the technician Christel Weyrauch is greatly appreciated.

\section{References}

Bernardi P, Forte M (2007) Novartis Found Symp 287:157-164

Bolender N, Sickmann A, Wagner R, Meisinger C, Pfanner N (2008) EMBO Rep 9:42-49

Bose M, Whittal RM, Gairola CG, Bose HS (2008) Toxicol Appl Pharmacol 227:284-290

Bowen KA, Tam K, Colombini M (1985) J Membr Biol 86:51-59

Brdiczka D, Beutner G, Rück A, Dolder M, Wallimann T (1998) Biofactors 8:235-242

Cheng EH, Sheiko TV, Fisher JK, Craigen WJ, Korsmeyer SJ (2003) Science 301:513-517
Desai MK, Mishra RN, Verma D, Nair S, Sopory SK, Reddy MK (2006) Plant Physiol Biochem 44:483-493

Dolder M, Zeth K, Tittmann P, Gross H, Welte W, Wallimann T (1999) J Struct Biol 127:64-71

Galluzzi L, Kepp O, Tajeddine N, Kroemer G (2008) Oncogene. In press

Grimm S, Brdiczka D (2007) Apoptosis 12:841-855

Guo XW, Smith PR, Cognon B, D'Arcangelis D, Dolginova E, Mannella CA (1995) J Struct Biol 114:41-59

Komarov AG, Deng D, Craigen WJ, Colombini M (2005) Biophys J 89:3950-3959

Koppel DA, Kinnally KW, Masters P, Forte M, Blachly-Dyson E, Mannella CA (1998) J Biol Chem 273:13794-13800

Malia TJ, Wagner G (2007) Biochemistry 46:514-525

Mannella CA (1998a) J Struct Biol 121:207-218

Mannella CA (1998b) J Struct Biol 121:207-218

Mazzoni C, Falcone C (2008) BBA 1783:1320-1327

Minetti CA, Tai JY, Blake MS, Pullen JK, Liang SM, Remeta DP (1997) J Biol Chem 272:10710-10720

Nahon E, Israelson A, Abu-Hamad S, Varda SB (2006) FEBS Lett 579:5105-5110

Pastorino JG, Hoek JB (2003) Curr Med Chem 10:1535-1551

Rostovtseva TK, Antonsson B, Suzuki M, Youle RJ, Colombini M, Bezrukov SM (2004) J Biol Chem 279:13575-13583

Salinas T, Duchêne AM, Delage L, Nilsson S, Glaser E, Zaepfel M, Maréchal-Drouard L (2006) Proc Nat Acad Sci 103:18362-18367

Schwertz H, Carter JM, Abdudureheman M, Russ M, Buerke U, Schlitt A, Müller-Werdan U, Prondzinsky R, Werdan K, Buerke M (2007) Proteomics 7:4579-4588

Shao L, Kinnally KW, Mannella CA (1996) Biophys J 71:778-786

Sukumaran S, Hauser K, Maier E, Benz R, Mäntele W (2006) Biochemistry 45:3972-3980

Yagoda N, von Rechenberg M, Zaganjor E, Bauer AJ, Yang WS, Fridman DJ, Wolpaw AJ, Smukste I, Peltier JM, Boniface JJ, Smith R, Lessnick SL, Sahasrabudhe S, Stockwell BR (2007) Nature 447:864-868

Young MJ, Bay DC, Hausner G, Court DA (2007) BMC Evol Biol $7: 31$ 